Erschienen in: Stickel, Gerhard (Hrsg.): Sprache - Sprachwissenschaft - Öffentlichkeit.

Berlin/New York: de Gruyter, 1999. S. 225-242.

(Jahrbuch des Instituts für Deutsche Sprache 1998)

DOI: https://doi.org/10.1515/9783110622645-015

\title{
Gisela SChOENTHAL
}

\section{Wirkungen der feministischen Sprachkritik in der Öffentlichkeit}

\begin{abstract}
Feministische Sprachkritik wird und wurde als sprachliches und sprachwissenschaftliches Thema in den letzen Jahren intensiv und folgenreich öffentlich diskutiert. Der Beitrag gibt einen Überblick über die Entwicklung in exemplarischen öffentlichen Lebensbereichen im deutschsprachigen Raum.
\end{abstract}

\section{Vorbemerkung ${ }^{1}$}

Die ersten deutschsprachigen Richtlinien gegen den sexistischen Sprachgebrauch wurden 1980 publiziert. Sie waren adressiert an alle, die ,professionell und offiziell geschriebene und gesprochene Sprache produzieren, vor allem an die, die - ob im Kindergarten, an der Schule oder in der Universität - Sprache lehren und an die, die in den Medien, in der Verlagsarbeit und anderswo Sprache verbreiten, an die Verfasserinnen und Verfasser von Lehr- und Fachbüchern, Berufsberatungstexten, Radio- und Fernsehtexten, Sachtexten, Wörterbüchern, Enzyklopädien, Werbetexte, Wettbewerbsausschreibungen, Zeitungs- und Zeitschriftenartikel jeglicher Art." (Guentherodt u.a. 1980, S. 15f.)

Wen haben sie erreicht? Eine Bilanz fällt nicht leicht, allein schon wegen des Umfangs an Aktivitäten und mittlerweile einschlägigen Publikationen. Die Entwicklung läuft in den einzelnen deutschsprachigen Ländern und den einzelnen Bundesländern zum Teil verschieden, ebenso in unterschiedlichen Kommunikationsbereichen, und sie variiert auch in $\mathrm{Ab}$ hängigkeit vom Medium und von den Aspekten der Kritik, ob es etwa um den Einsatz des Binnen-I oder von Schrägstrichen geht. Ich beobachte diese Entwicklung seit mehr als zwanzig Jahren (Schoenthal 1985, 1989, 1998) und praktiziere selbst ziemlich weitgehend feministische Sprach-

I Der Text wurde unter wesentlicher Beibehaltung der Vortragsform geringfügig überarbeitet. 
kritik. Ich glaube nicht, daß Sprache das Wichtigste ist, glaube aber auch nicht, daß Sprache sich von den anderen Aspekten der gesellschaftlichen Wirklichkeit trennen läßt, sie ist nicht zentral, aber immer beteiligt, immerzu sprechen wir über Menschen, immer wieder müssen Texte gestaltet, Verträge formuliert oder übersetzt werden. Eine vor allem negative Bilanz der zahlreichen Kritik an der feministischen Sprachkritik hat wiederholt Marlis Hellinger vorgelegt, zuletzt 1997. Mein Anliegen ist eine positive Bilanz der Wirkungen, die sie bisher erzielt hat. Dafür stütze ich mich auch auf Publikationen aus einem soeben erschienenen, von mir herausgegebenen Sammelband zum Thema (Schoenthal 1998).

Luise Pusch hat 1984 den feministischen Sprachgebrauch dokumentiert, 1998 zeigt sich: bestimmte Formen, die sie damals dokumentiert hat, waren Adhocbildungen, andere spielen am Rande eine Rolle. Es geht um die Personenbezeichnungen: im Mittelpunkt stehen Wörter mit den Endungen -in, -frau zur Bezeichnung von Frauen; außerdem geht es um Paarformen (auch Splitting genannt) sowie die Möglichkeiten des geschlechtsneutralen Sprachgebrauchs für die Bezeichnung gemischter Gruppen bzw. Personen oder Gruppen unbekannten Geschlechts. Neu gegenüber Pusch (1984) ist das Binnen-I.

\section{Exemplarische Einflußbereiche feministischer Sprachkritik}

Ich habe für meine Bilanz exemplarisch wichtige Einflußbereiche der feministischen Sprachkritik ausgewählt, und werde-auch hier exemplarisch und in sehr unterschiedlicher Ausführlichkeit erläutern, was sich tut, getan hat.

\subsection{Verwaltungs- und Gesetzessprache}

Grundsätzlich ist klar, daß Juristen und Juristinnen zuständig sind, und das Problem aufgreifen und diskutieren müssen, ob Frauen durch die Sprache unsichtbar gemacht werden, ob Frauen benachteiligt werden. Es gab ja auch schon vor der feministischen Sprachkritik Gesetzesänderungen, die maskuline Personenbezeichnungen durch neutrale ersetzt haben, damit Frauen nicht sprachlich ausgeschlossen sind. Beispielsweise wurde im Familienrecht das neutrale Wort Elternteil eingefuihrt.

Mitte der 80er Jahre geriet das Thema, das zunächst durch Senta Trömel-Plötz und dann vor allem durch Luise Pusch in engagierter Vortragsund Publikationstätigkeit zu einem feministischen und linguistischen Thema mit großer Resonanz geworden war, in den Kontext der Politik. Der Textausschnitt 1 aus der ,Zeit" von 1987 im Anhang ist für diese Diskussion ein Schlüsseltext: auf Antrag der SPD und der Grünen wurde in sämtlichen Parlamenten eine Diskussion über Sprachveränderungen geführt: erstes Beispiel im Bundestag war der Wahlmännerausschuß, ein 
Gremium, in dem Frauen sich sprachlich nicht wiederfinden wollten und konnten. Rita Süßmuth, eine gebildete Politikerin und Mitglied einer konservativen Partei, macht das Anliegen der Sprachveränderung zu ihrem eigenen. In diesem Text entfaltet sie das Beispiel vom Arzt, der im Praktikum schwanger wird, als einen Beleg für den kritisierten Sprachgebrauch.

Inzwischen ist es in allen deutschsprachigen Ländern zu einer institutionalisierten Veränderung in Richtung der Forderungen der feministischen Sprachkritik gekommen. Im Bereich der Verwaltungssprache sind eine Vielzahl von Texten, Formularen, Vordrucken, Muster von Urkunden, Haushaltsplänen in Behörden geschlechtergerecht gestaltet. Sie alle kennen selbst genügend Beispiele. Bei der Volkszählung 1987 hat man alle Fragebögen eingestampft und neu so gestaltet, daß Frauen sich angesprochen fühlten, aus Angst, sie würden sonst die Befragung verweigern. Im Personalausweis heißt es mittlerweile: Unterschrift des Inhabers/ der Inhaberin und im Paß von Frauen steht: Die Inhaberin dieses Passes ist eine Deutsche. In vielen Bereichen und Institutionen wurden Richtlinien, Anleitungen, Leitfäden erarbeitet und umgesetzt, die helfen von der unproduktiven Kritik zu produktiven, phantasievollen sprachlichen Lösungen zu gelangen. Das ist vor allem auch ein Verdienst der vielen Frauenbeauftragten, die seit Mitte der 80er Jahre eingestellt wurden. Einer der bekanntesten und gelungensten ist sicher der Leitfaden der schweizerischen Bundeskanzlei aus dem Jahr 1996. Inzwischen liegt auch eine neue österreichische Version vor (Kargl. u. a. 1997, nach Wodak u. a. 1987). Beide Länder ziehen vor zu empfehlen, nicht vorzuschreiben, beide Texte propagieren die kreative Lösung. Wer in diesen Nachschlagewerken blättert, wird schnell von der Sorge befreit, es würden nur umständlichere und unökonomische Texte entstehen.

Auf beträchtliche Schwierigkeiten stieß und stößt aber die Umgestaltung der Gesetzessprache. Die Diskussion ist in Deutschland noch nicht abgeschlossen. Die Beibehaltung des genetischen Maskulinums als alleinige Lösung, wie Stickel (1988, S. 352) vorgeschlagen hat, hat sich nicht durchgesetzt. Der Bund und einige Bundesländer haben unterschiedliche Regelungen getroffen, die alle das geschlechtsneutrale Formulieren betonen (vgl. Frank-Cyrus/Dietrich 1998). Nur auf diese Weise sehen sie neben der grundsätzlichen sprachlichen Gleichbehandlung auch die anderen sprachlichen Grundsätze für die Gestaltung von Rechtsvorschriften wie Allgemeinheit, Knappheit, Verständlichkeit und Klarheit gewahrt. Aber bei Regelungen in Zusammenhang mit Mutterschutz, Schwangerschaft etc. dürfen genetische Maskulina nicht verwendet werden (Arbeitsgruppe Rechtssprache 1991, S. 37) Auch die in der Diskussion engagierten Feministinnen fordern keine umfassende Feminisierung der Rechtstexte, auch sie wollen das genetische Maskulinum durch neutrale Bezeichnungen ersetzen in Gesetzen, die von der Gleichstellung der Frau 
nicht betroffen sind. Verwendung des Femininums in der Paarbezeichnung hingegen fordern sie in Normen, die die defizitäre gesellschaftliche Stellung der Frau widerspiegeln oder fortzusetzen geeignet sind (Grabrucker 1993, S. 226). Die Gesellschaft für deutsche Sprache hat im Auftrag der interministeriellen Arbeitsgruppe Rechtssprache des Deutschen Bundestages Empfehlungsschriften und Erlasse verglichen (Frank-Cyrus/Dietrich 1998). Nach ihrer Auffassung wird ein Modell zur Umgestaltung von Rechtsvorschriften, das geschlechtsneutrale Formen, die Paarform und das genetische Maskulinum verbindet, am ehesten den komplexen Anforderungen der Rechtssprache gerecht. Die Paarform bietet sich für ,personennahe" Formulierungen an, bei juristischen Personen und abstrakten Funktionen hingegen kann das genetische Maskulinum beibehalten werden. Im Auftrag des Bundesjustizministeriums hat die Gesellschaft für deutsche Sprache inzwischen auch eine Meinungsbefragung durchgeführt (Frank-Cyrus/Dietrich 1997, vgl. auch 4.), aus der sich ergibt, daß $80 \%$ der Befragten eine geschlechtergerechte Veränderung von Gesetzestexten wünschen. Einzelne veränderte Gesetze gibt es in allen deutschsprachigen Ländern: dazu gehört das 1985 geänderte viel zitierte Hebammengesetz, das nur für einige Personenbezeichnungen Paarformen einführte (Schüler und Schülerin, Arzt und Arztin; vgl. Schoenthal 1989). 1994 hat in Österreich im ArbeitnehmerInnenschutzgesetz sogar das Binnen-I Gesetzeskraft erlangt (Kargl u. a., S. 133)

Einen besonders prominenten Platz im Rahmen der Verwaltungs- und Gesetzessprache nehmen die Berufsbezeichnungen ein, wie auch Süßmuth betont; Pusch hat darauf hingewiesen, daß in der Duden-Grammatik von 1954 ein Beispielsatz notiert ist: Grete B. ist Rechtsanwalt (mit dem Hinweis in Klammern: auch schon Rechtanwältin). Mittlerweile haben sich die movierten Berufsbezeichnungen, gegen die Wustmann schon um die Jahrhundertwende nichts einzuwenden hatte, in starkem $\mathrm{Maße}$ durchgesetzt. Dabei hat gerade die Verwaltungssprache auch eine wichtige Funktion für die Alltagssprache: in Berufsverzeichnissen wurden movierte Formen schon in den 70er Jahren eingefuihrt, um jungen Frauen ein breiteres Berufsspektrum bewußt zu machen.

Ich habe im Anhang als Text 2 zwei Todesanzeigen gegenübergestellt, die diese sprachliche Entwicklung dokumentieren: die Gestorbene legte altersbedingt Zeit ihres Lebens Wert darauf, Rechtsanwalt zu sein, so ist klar, daß ihre eigene Kanzlei diesen Sprachgebrauch auch beibehält. Die Anzeige der Rechtsanwaltskammer vom nächsten Tag folgt selbstverständlich dem inzwischen üblichen Sprachgebrauch.

Das Beispiel Berufsbezeichnungen zeigt besonders deutlich, in welchem Maße die Gesetzes- und Verwaltungssprache mit der Alltagssprache verzahnt ist, und wie sich das dann wieder in den Medien spiegelt. Allmählich bahnt sich auch bei den movierungsresistenten Titeln und Graden 
eine Änderung an: in Österreich ist ihre Verwendung seit 1993 gesetzlich geregelt, in Deutschland fangen Frauen an, sich die Titel gerichtlich zu erstreiten, je nach Bundesland geht das auch ohne juristische Hindernisse.

Berufsbezeichnungen sind auch ein ganz wichtiger Bestandteil von Stellenanzeigen (vgl. 2.3.). Schon seit 1980 gibt es in der Bundesrepublik eine Sollvorschrift, die regelt, daß Arbeitsplätze ${ }^{2}$ geschlechtsneutral auszuschreiben sind, inzwischen, seit 1997 übrigens eine Mußvorschrift. Diese hatte bisher vor allem Konsequenzen für den öffentlichen Dienst. Aus einer im März 1998 veröffentlichten Untersuchung des Stellenmarkts der nordrhein-westfälischen Gleichstellungsministerin geht hervor, daß jede zweite der untersuchten 3275 Anzeigen gegen das geltendes Recht der geschlechtsneutralen Arbeitsplatzausschreibung verstieß: Anzeigen für höhere Positionen wandten sich z. B. häufig nur an Männer, umgekehrt richteten sich im sozialen und als frauentypisch geltenden Bereich die Anzeigen nur an weibliche Stellensuchende (vgl. Bad. Zeitung vom 7.3.1998).

\subsection{Theologie}

Neben Juristinnen und Juristen sind auch Theologen und Theologinnen von berufswegen angesprochen, wenn es darum geht, Ungleichbehandlung aufzudecken oder abzubauen.

Auch die Evangelische Landeskirche Baden-Württemberg hat sich dieses Anliegen zu eigen gemacht. Ich habe als Text 3 im Anhang deshalb Vorwort und Einleitung aus der dort verfaßten kleinen Broschüre „Empfehlungen für eine Sprache, die Frauen anspricht", abgedruckt, stellvertretend für die Vielzahl von Richtlinien und Anleitungen, die mittlerweile meist für den internen Gebrauch in Institutionen entstanden sind. Hier wird kein Zweifel daran gelassen, daß Sprache das Bewußtsein formt.

Vor allem feministische Theologinnen stellen die feministische Sprachkritik in den Kontext des gerechten Sprechens über alle Gruppen (Behinderte, Farbige ...). Feministische Theologinnen sind es auch, die eine frauengerechte Übersetzung von einseitigen und falschen Formulierungen in Bibeltexten fordern. „Frauen wollen hören, daß es Prophetinnen, Jüngerinnen, Gemeindeleiterinnen und Apostelinnen gab." (Wegner u. a. 1990, S. 95) Feministische Theologinnen fordern darüber hinaus auch eine veränderte Metaphorik in Gebeten und Liedern: Gott soll nicht nur auf männliche Bilder wie Vater, Herr, König, Hirte, Heiliger Geist reduziert sein (Wegner u. a. S. 190, S. 151). Ich erwähne das als einen wichtigen Aspekt feministischer Sprachkritik; ich betone aber auch, daß dieser Aspekt sehr

2 Die Frage, ob movierte Formen für Frauen zu verwenden seien, wurde auch in der DDR ausführlich diskutiert, erstaunlicherweise schon in den 50er Jahren, vgl. dazu Diehl (1992). 
sorgfältig von der formalen Gestaltung von Wörtern, Sätzen und Texten zu trennen ist.

\subsection{Medien}

Durch die Diskussion in politischen Gremien ist seit Ende der 80er Jahre die feministische Sprachkritik in die Schlagzeilen geraten, ein besonders eindrucksvolles Beispiel fand sich in der Münchner Abendzeitung vom November 1990 (vgl. Anhang Text 4). Vor allem konservative Printmedien haben in Glossen und Kommentaren feministische Sprachkritik massiv kritisiert und das Anliegen herabgewürdigt, ein Aspekt, den ich hier nur erwähnen, nicht ausführen will. Noch immer läuft einiges in die Richtung die auch Süßmuth nennt: Wie können wir es am besten lächerlich machen? Legitimation der feministischen Sprachkritik ist nicht mein Thema. Ich verweise dazu auf die Darstellungen von Hellinger, außerdem auf Hildegard Gorny (1995), die mit vielen schönen Zitaten die Berichterstattung in den Medien über sprachpolitische Aktivitäten dokumentiert hat.

Bei Printmedien wurde bisher meist der Sprachgebrauch in Stellenanzeigen untersucht (vgl. auch 1.). Hier kann man auch gesetzesbedingt eine Entwicklung feststellen, Frauen mehr sichtbar zu machen, die aber eben längst nicht alle Anzeigen trifft.

Ursula Doleschal (1998) hat für Österreich herausgefunden, daß abgesehen von den Stellenanzeigen die Tageszeitungen „Standard“ und „Presse" Tendenzen im Sinne der feministischen Sprachkritik fast nur auf Initiative einzelner JournalistInnen und GastkommentatorInnen zeigen. Die als kritisch geltende Wochenzeitung „Profil“ läßt mehr Raum für innovativen Sprachgebrauch, eine Blattlinie gibt es aber nicht. Auch Ann Peyer und Eva Lia Wyss (1998) beobachten, daß die Schweizer überregionalen Zeitungen wie „NZZ“, „Tagesanzeiger“ und „Weltwoche“ bei der Umsetzung der feministischen Sprachkritik vorsichtig sind. Und Gisela Trempelmann (1998) hat beim Vergleich der beiden korrespondierenden Zeitungen „Potsdamer Neueste Nachrichten“ (PNN) und „Der Tagesspiegel“ (Westberlin) festgestellt, daß die Westberliner Zeitung in Anrede und Personenbezeichnung die Paarformen verwendet (Liebe Leserinnen und Leser), die PNN hingegen nicht.

Wenn ich meine eigenen Lektüreerfahrungen und -beobachtungen dazu nehme, läßt sich zusammenfassend sagen, daß zur Bezeichnungen von Frauen in der Regel movierte Formen verwendet werden, zur Bezeichnung gemischter Gruppen oder Personen unbekannten Geschlechts hingegen dominiert das geschlechtsneutrale Maskulinum in fast allen Zeitungen. Vereinzelt finden sich allerdings immer Texte, denen anzumerken ist, daß sie mit den Grundgedanken der feministischen Sprachkritik im Hinterkopf formuliert wurden; es setzt jedoch eine sehr aufmerksame Lektüre voraus, Texte dieser Art aufzuspüren, wobei allerdings die Themenschwerpunkte 
oft eine Hilfe sind. Solche Texte stehen immer wieder in der Badischen Zeitung, die ich täglich lese. Erstaunlich wenig bemühen sich die meisten Frauenzeitungen um eine Frauen sichtbar machende Sprache. Einzelformulierungen, die eine feministisch sprachkritische Reflexion erkennen lassen, finden sich immer wieder in allen Zeitungen - frau, mann, mensch, Eltern bzw. Väter und Mütter des Grundgesetzes ... Auffällig ist zum Beispiel bei Interviews, daß die Zeitungen den Sprachgebrauch der angesprochenen Gruppe übernehmen, und entgegen den sonstigen Sprachgebrauch zum Beispiel von Studierenden statt von Studenten schreiben.

Wirkliche sprachliche Innovationen, die Frauen sichtbar machen, finden sich vor allem in der Regional- und Alternativpresse: so war die taz deutsche Vorreiterin für das große I. Im Gegensatz zur Schweizer woz, von der sie den Sprachgebrauch in den 80er Jahren übernommen hatte, findet sich in der taz das große I nicht mehr so häufig. Nach telephonischer Auskunft aus Berlin, ist den VerfasserInnen der Artikel die Handhabung überlassen. Der Sprachgebrauch der elektronischen Medien in seiner Vielfalt ist nahezu noch nicht untersucht worden. Doleschal (1998) sieht für Österreich wenig Unterschiede zu den Printmedien, und wie so oft ist auch hier die Schweiz Vorreiterin: bei öffentlich-rechtlichen und lokalen Rundfunksendern ist den ModeratorInnen der Sprachgebrauch nicht freigestellt: es gibt interne Richtlinien, für Personenbezeichnungen die Paarformen zu verwenden, sowohl bei Mundart als auch bei Standardsprache (Peyer/Wyss 1998).

\subsection{Belletristik und Fachliteratur}

In der schöngeistigen Literatur sind die Innovationen feministischer Sprachkritik nicht rezipiert worden. Sie ist aber ein Bereich, in dem sehr konsequent und gelegentlich originell und innovativ moviert wird und damit eigentlich die spielerisch-kreative Seite der feministischen Sprachkritk aufgegriffen wird. Ein besonders markierter Text aus der Hochzeit des Feminismus ist Günther Grass' Endzeitroman „Die Rättin“ (1986). Der Titel zeichnet sich durch das demonstrative, da von einem Femininum Ratte abgeleitete Femininum aus, analog sind etwa auch die Formen Täubin, Kätzin belegt. In der Regel werden ja feminine Movierungen von Maskulina und, bei Menschen extrem selten, bei Tieren aber häufiger, maskuline Movierungen von Feminina abgeleitet. Ratte als Oberbegriff und genetischer Ausdruck wird aber von Grass offenbar nicht als ausreichende Feminisierung empfunden. Wichtige Akteurinnen des Romans sind neben der Rättin fünf Frauen mit zum Teil recht ungewöhnlichen Berufen, aber selbstverständlich moviert verwendeten Berufsbezeichnungen Kapitänin, Steuermännin, Maschinistin, Meereskundlerin und Köchin einer Schiffsmannschaft. Wie der Autor die Rättin, die fünf Frauen, den Erzähler und sich selbst damit im Feminismus verorten will, kann hier in seiner 
Vielschichtigkeit nicht diskutiert werden. Die Wahl der Berufsbezeichnung Steuermännin kennzeichnet den Text sehr deutlich als einen der achtziqer Jahre, heute hätte Grass sich wohl für Steuerfrau entschieden. Bei der Schweizer Autorin Eveline Hasler finde ich in ihrem Buch „Die Wachsflügelfrau" aus dem Jahr 1995 die movierten Formen Kolossin und Menschin. ${ }^{3}$

Movierungen haben in der Literatursprache der DDR einen anderen Stellenwert: vor dem Hintergrund des öffentlichen Sprachgebrauchs in dem konsequent nicht moviert wurde, (Margot Honecker war selbstverständlich Volksbildungsminister) erweisen sich Movierungen als subversives Sprechen. Beate Schröter (1994, S. 131) beobachtet in den von ihr untersuchten Romanen der Jahre 1945-1965 sowie der Jahre 1968-1989, daß Schriftstellerinnnen und Schriftsteller bis auf wenige Ausnahmen die movierte Form verwenden. Auch Trempelmann (1990) konstatiert einen Prozeß der Normveränderung, der abgelehnt, aber nicht aufgehalten werden kann. Am Beispiel des Romans von Erwin Strittmatter „Der Laden“ (1983) dokumentiert sie poetische Neu- oder Einmalbildungen auf -in wie Menschin, Lieblingin, Liebstin, Gästin, Jemandin, sowie eine Reihe von Komposita, wie Gesellinnenstück, Gefährtinnenschwarm, Schneiderinnenspiegel und Schülerinnenrespekt (1990, S. 38) „wie sie sich Feministinnen nicht schöner hätten ausdenken können."

Wichtig ist natürlich der Kontext der Ableitung: nur ihm kann ich entnehmen, ob sie ernsthaft, wenn auch spielerisch oder ironischkritisch zu verstehen ist.

Andere Elemente der feministischen Sprachkritik bis hin zum Binnen-I habe ich nur in Übersetzungen feministischer Kriminalromane aus dem Englischen gefunden. Ebenso gilt auch für fachsprachliche Texte gerade im Bereich der Geistes- bzw. Sozialwissenschaften, daß Paarformen und groBes I mit erstaunlicher Häufigigkeit und Konsequenz verwendet werden.

\subsection{Schule und Hochschule}

In der Korrespondenz des Gymnasiums meiner Kinder fallen mir über all die Jahre immer wieder inkonsequente Texte auf, bestimmte Personenbezeichnungen werden als Paarform verwendet aber nicht alle. Zwar heißt es zum Beispiel in der Anrede: Liebe Kolleginnen und Kollegen, aber Liebe Schüler. Das kann Ausdruck der Tatsache sein, daß die Sprachveränderungen nur halbherzig betrieben werden, aber auch der Tatsache, daß diese Sprachänderungen Aufmerksamkeit und Nachdenken erfordern und ihr Gebrauch eben längst nicht automatisiert ist.

3 Eine lesenswerte Sammlung von originellen und schönen Beispielen aus der deutschsprachigen Literatur vor aller feministischen Sprachkritik findet sich bei Ljungerud (1973). 
Meiner Erfahrung nach ist vor allem im Bereich der Erwachsenenbildung vielen lehrenden Frauen der Gedanke „Frauen sollen nicht nur mitgemeint sein" sehr wichtig. Hier sind weitere Untersuchungen notwendig, sicher spielen eine ganze Reihe Faktoren eine Rolle bis hin zur politischen Ausrichtung der jeweiligen Landesregierung.

Schule ist wie feministische Theologie auch ein ganz wichtiger Bereich der Textkritik geworden: Schulbücher enthalten in allen Fächern fiktionale Texte. Dazu gibt es mittlerweile eine sehr umfangreiche Literatur, deren zentrale Aussage ist: Frauen und Mädchen kommen nicht vor, d. h. es wird nicht nur die fehlende Movierung der Personenbezeichnugen beklagt, sondern vor allem, daß die Beispiele und kleinen Texte nur Männer und Jungen thematisieren oder wenn Frauen und Mädchen, dann nur in absolut klischeeverhaften Darstellungen. Im Bereich Schule sind diese Texte noch längst nicht aus dem Verkehr gezogen. Auch hier möchte ich diesen Aspekt nur erwähnen.

Wie vielen von Ihnen aus Wandzeitungen und Seminaren sicher geläufig ist, ist im schriftlichen und mündlichen universitären Sprachgebrauch die Forderung, Frauen sichtbar zu machen, mindestens aber, Frauen und Männer gleichzubehandeln, am häufigsten verwirklicht, allerdings auch hier weit entfernt von jeder Durchsetzung und auch hier sicher abhängig von der Sprachpolitik der jeweiligen Landesregierung.

Doleschal (1998) hat das für die Universität Wien genauer untersucht. Nach ihrere Beobachtung verläuft die Entwicklung nicht einheitlich sondern in Abhängigkeit von politischen Ereignissen. Außerdem erfolgt etwa die Verwendung des Binnen-I oft nicht zum Zwecke des Ausdrucks sprachlicher Gleichbehandlung, sondern dient dem Ausdruck der Ingroup-Zugehörigkeit. Karin Wetschanov (1997) zeigt am Beispiel einer links-alternativen Gruppe, daß selbst hier Veränderungsansprüche und tatsächliches Kommunikationsverhalten auseinanderklaffen: das Binnen-I ist keinesfalls internalisiert.

\section{Das Binnen-I}

Das Binnen-I ist wohl die sprachliche qualitative Innovation, die die meisten Sprachangehörigen mit der feministischen Sprachkritik verbinden. Sein Einsatz und seine Verbreitung verdienen noch umfangreiche Forschung. Nach Meinung von Doleschal (1998) wird es fakultativ verwendet, außerdem scheinen bestimmte Wörter auch verwendungsanfälliger zu sein als andere, etwa MitarbeiterInnen. Ich habe es im Zusammenhang mit den bisher behandelten Aspekten schon mehrfach erwähnt und zähle deshalb nur noch ein paar Beispiele auf, die die Spannne von Ablehnung bis Akzeptanz im öffentlichen Leben illustrieren.

- In Baden-Württemberg hat die Ministerin seinen Gebrauch in den Schulen verboten. 
- Im Gebiet der ehemaligen DDR hat es im öffentlichen Leben seit der Wende beträchtliche Verbreitung erlangt.

- Der Leitfaden der schweizerischen Bundeskanzlei empfiehlt es für verknappte, nicht aber für ausformulierte fortlaufende Texte.

- In Osterreich hat es 1994 im ArbeitnehmerInnenschutzgesetz sogar Gesetzeskraft erlangt (Kargl u. a. S. 133).

- Selbst Sabine Etzold von der Wochenzeitung „Die Zeit“ kann ihm eine gewisse zähneknirschende Anerkennung nicht versagen (Etzold 1996).

\section{Meinungsbefragungen}

Aus den letzten zehn Jahren gibt es eine ganze Reihe mehr oder weniger umfangreicher Meinungsbefragungen, das sind in Österreich Wodak u. a. (1987); in der Schweiz SPRACHE MACHT POLITIK (1994) und Peyer/Wyss (1998), im universitären Kontext in Deutschland: Gansel (1995); Alfers u. a. (1994); zum großen I in der ehemaligen DDR Trempelmann (1998); Frank-Cyrus/Dietrich (1997) zur Gesetzessprache. Ich gehe zunächst auf die Befragung zur Gestaltung der Gesetzessprache ein (Frank-Cyrus/Dietrich 1997), eine Befragung der Gesellschaft für deutsche Sprache im Auftrag des Bundesministeriums für Justiz. Den Fragebogen haben vorwiegend Personen jüngeren bis mittleren Alters ausgefüllt. Frauen sind besonders stark vertreten. Die Befragten haben überwiegend eine sehr gute Bildung und sind meist in guter beruflicher Stellung tätig, Studierende sind stark beteiligt.

Beim Vergleich der vorgelegten verschiedenen Versionen von Gesetzestexten tendieren die befragten Männer zu Veränderungen in Richtung geschlechtsneutraler Formulierungen; die Frauen zu Paarformen. Die Originalfassung findet bei beiden Geschlechtern wenig Zustimmung, $d . h$. fast $80 \%$ treten für eine geschlechtergerechte Umgestaltung von Gesetzestexten ein. Interessant gerade für die Diskussion im Rahmen dieser Jahrestagung ist außerdem: Personen mit sprachbezogenen Berufen in den Medien sind eher für geschlechtsneutrale Formulierungen, Personen mit pädagogischen Berufen sind eher für Paarformen.

In der Gemeindeordnung von Wädenswil war das genetische Femininum (Politikerinnen anstelle von Politiker und Politikerinnen) per Beschluß des Parlaments eingeführt worden, bei der Volksabstimmung nach intensiv geführter Auseinandersetzung aber abgelehnt worden. Unmittelbar nach der Abstimmung wurde die sozialwissenschaftliche Studie SPRACHE MACHT POLITIK (1994) zum Zusammenhang zwischen politischer und sprachlicher Gleichstellung durchgeführt. Durch diesen politischen Zusammenhang wurden auch Personen mit dem Thema konfrontiert, die sich normalerweise nicht für Sprache interessieren. Das Ergebnis ist nach Peyer/Wyss (1998): Zwar finden rund 80\% der Befragten, es gebe 
wichtigere Probleme als die sprachliche Gleichstellung, dennoch geben etwa gleich viele an, daß sie sich in ihrer eigenen Praxis darum bemühen. Überdurchschnittlich groß ist der Anteil an Frauen aus der mittleren Altersgruppe (26-45 Jahre), an Frauen mit höherer Schulbildung und an erwerbstätigen Frauen. Außerdem zeigt sich eine enger Zusammenhang zwischen Zustimmung zu politischer und zu sprachlicher Gleichstellung.

Die Meinungsbefragungen insgesamt zeigen ein beträchtliches Interesse am Thema, was nicht heißt, daß die Personen Sprachkritik besonders wichtig finden und auch nicht, daß sie den Forderungen immer folgen. Eine hohe Prozentzahl bemüht sich aber in der eigenen Praxis darum.

\section{Schluß}

Kein sprachpolitisches Thema hat bisher vergleichbaren Erfolg und Wirkungen erzielt. Welches sind diese Wirkungen?

Feministische Sprachkritik ist eines der wenigen sprachlichen und sprachwissenschaftlichen Themen, das öffentlich intensiv diskutiert wurde und beträchtliche Einstellungsänderungen gegenüber dem Sprachgebrauch ausgelöst hat. Der Höhepunkt der Diskussion ist überschritten, Umsetzung, Institutionalisierung stehen in Vordergrund.

Niederschlag in der öffentlichen Praxis heißt weitgehende Durchsetzung der vorher schon praktizierten Movierung, als quantitative Veränderung für viele Frauen einfach eine Selbstverständlichkeit. Darüber hinaus gibt es, je nach Kommunikationsbereichen verschieden, Tendenzen in Richtung Vielfalt der möglichen Formen, allerdings bei grundsätzlicher Beibehaltung des genetischen Maskulinums.

Erstaunlich ist, in welchem Ausmaß sich die Verwendung von Paarformen, die selbst von Pusch (1984) als unökonomisch empfunden wird, in der Schweiz durchgesetzt hat.

Gewinnerinnen sind die Verwaltungssprache, in Ansätzen auch Gesetzessprache, weiter Sprache und Kommunikation im universitären Bereich, die Alltagssprache, am wenigsten noch die Mediensprache mit Ausnahme der Anzeigen. Weitere Einflußbereiche sind noch eingehend zu untersuchen: zum Beispiel mündliche und schriftliche Kommunikation in politischen Parteien, Schulen, elektronischen Medien.

Die Entwicklung geht in einzelnen Ländern verschieden weit, ganz sicher ist Deutschland nicht mehr federführend, wie noch in den 80er Jahren.

Die Sprachänderungen sind partiell Unterscheidungskriterien zwischen Deutschland Ost und Deutschland West „Wessideutsch" (Trempelmann 1998).

Von allen Änderungen, die durch die feministische Sprachkritik initiiert oder forciert worden sind, gehört die Verwendung femininer Formen zur Bezeichung von Frauen zu den am stärksten automatisierten. Für alle 
möglichen Kontexte ist der Sprachgebrauch immer noch schwankend in Abhängigkeit davon, ob es sich um eine öffentliche oder eine private Situation handelt, geschrieben oder gesprochen wird, ob reflektiert oder unreflektiert formuliert wird und anderen Faktoren mehr. Deshalb ist selbstverständlich gerade auch in der Alltagssprache immer wieder zu hören, daß Frauen sagen: ich als Lehrer, Referendar, Nichtraucher ... Es bleibt abzuwarten, wie sich das weiterentwickelt.

Feministische Sprachkritik hat mich gelehrt, Kreativität im Umgang mit der Sprache als wichtiges Ziel an Germanistikstudierende zu vermitteln für Schule und andere Bereiche.

Den Ausdruck 'political correctness' akzeptiere ich dafür nur, wenn darunter verstanden wird, Frauen und Männer gleichzubehandeln, Frauen nicht mehr und nicht anders als Männer abzuwerten, nicht aber wenn darunter haupsächlich verstanden wird, Frauen aufzuwerten, wie die Darstellung von D. E. Zimmer (1997) suggeriert. ${ }^{4}$ Eine solche Beschreibung wird der feministischen Sprachkritik nicht gerecht.

Feministische Sprachkritik vermittelt auch die Erfahrung, daß Sprache verändert werden darf. Solche Änderungen werden ständig vorgenommen, häufiger als das im Alltag bewußt ist auch von Gesetzes wegen, hierzu gehört zum Beispiel das mir unverständliche gesetzliche Verbot des Wortes Hebammmer (Behn 1980).

Sprachwandel von oben passiert ständig: der besondere Erfolg der feministischen Sprachkritik beruht darauf, daß er von unten ausgelöst und forciert wurde.

\section{Literatur}

Alfers, Sandra/Kürschner, Wilfried/Pelka, Christiane (1994): Sprachsexismus? Die Bezeichnung der Geschlechter in der Sprachpraxis. In: van Laer, Hermann/Schmitt von Mühlenfels, Astrid (Hg.): Frauenfragen - Frauensache. Cloppenburg. S. 247-273.

Arbeitsgruppe Rechtssprache (1990): Maskuline und feminine Personenbezeichnungen in der Rechtssprache. Bundestagsdrucksache Bonn, 12/1041 vom 7.8.1991.

Behn, Michael (1980): „Neue“ Ausbildungsberufe. In: Zs.f. Sozialreform 26, S. $375-382$.

Diehl, Elke (1992): Ich bin ein Student. In: Deutschland Archiv 1992, S. 384-392.

Doleschal, Ursula (1998): Entwicklungen und Auswirkungen der feministischen Sprachkritik in Österreich seit 1987. In: Schoenthal (Hg.)

4 Ein Beispiel für political corectness in meinem Sinne: Am 22.2.1998 ist die Hess. Umweltministerin zurückgetreten. Vorgeworfen wird ihr, salopp formuliert: „Cousinenwirtschaft", ein Ausdruck, der neu ist, aber den vorgeworfenen Tatbestand alltagssprachlich korrekt wiedergibt. Die lexikalische Entsprechung zu Vetter ist Cousine, da es sich nur um Frauen handelt, ist dies unzweifelhaft der richtige Ausdruck. 
Etzold, Sabine (1996): Das Binnen-I west überall. In: DIE ZEIT Nr.15, S. 33.

Frank-Cyrus, Karin/Dietrich, Margot (1997): Sprachliche Gleichbehandlung von Frauen und Männern in Gesetzestexten. In: Der Sprachdienst 41, S. 55-68.

Frank-Cyrus, Karin /Dietrich, Margot (1998): Gesetze geschlechtergerecht gestalten - aber wie? Zwei Gutachten der Gesellschaft für deutsche Sprache für das Bundesministerium der Justiz. In: Schoenthal (Hg.).

Gansel, Christina (1995): Sprachwandel und Feminismus. Anspruch und „Wirklichkeit". In: DU 48, S. 322-328.

Gorny, Hildegard (1995): Feministische Sprachkritik. In: Stötzel, Georg/Wengeler, Martin (Hg.): Kontroverse Begriffe: Geschichte des öffentlichen Sprachgebrauchs in der Bundesrepublik Deutschland. Berlin/New York. S. 517-562.

Guentherodt, Ingrid/ Hellinger, Marlis/Pusch, Luise F./Trömel-Plötz, Senta (1980): Richtlinien zur Vermeidung sexistischen Sprachgebrauchs. In: LB69, S. 15-21.

Hellinger, Marlis/Schräpel, Beate (1983): Über die sprachliche Gleichbehandlung von Frauen und Männern. In: JiG, S. 40-69.

Hellinger, Marlis (1997): The discourse of distortion. Political correctness and feminist language reform. In: Braun, Friederike / Pasero, Ulrike ( $\mathrm{Hg}$.): Kommunikation von Geschlecht - Communication of gender. Pfaffenweiler. S. 164-182. (dt. in: MU 1996)

Kargl, Maria/Wetschanov, Karin/Wodak, Ruth (Mitarbeit: Nöla Perle) (1997): Kreatives Formulieren. Anleitungen zu geschlechtergerechtem Sprachgebrauch. Wien. (Schriftenreihe der Frauenministerin Band 13).

Ljungerud, Ivar (1973): Bemerkungen zur Movierung in der deutschen Gegenwartssprache. In: Linguistische Studien III. Hg. von Hugo Moser et al. Düsseldorf. S. 145-162.

Müller, Sigrid/Fuchs, Claudia (1993): Handbuch zur nichtsexistischen Sprachverwendung in öffentlichen Texten. Frankfurt.

Peyer, Ann/Wyss, Eva Lia (1998): „JazzmusikerInnen weder Asketen noch MüsliFif is". Feministische Sprachkritik in der Schweiz, ein Überblick. In: Schoenthal (Hg.).

Von Polenz, Peter (1982): Sprachkritik und Sprachnormenkritik. In: Heringer, Hans Jürgen (Hg.): Holzfeuer im hölzernen Ofen. Tübingen. S. 70-93. (erstmalig 1973)

Pusch, Luise F. (1984): Das Deutsche als Männersprache. Aufsätze und Glossen zur feministischen Linguistik, Frankfurt.

Schoenthal, Gisela (1985): Sprache und Geschlecht. In: Deutsche Sprache 13, S. $143-185$.

Schoenthal; Gisela (1989): Personenbezeichnungen im Deutschen als Gegenstand feministischer Sprachkritik. In: ZGL 17, S. 296-314.

Schoenthal, Gisela (1998): Von Burschinnen und Azubinnen. Feministische Sprachkritik in den westlichen Bundesländern. In: Schoenthal ( $\mathrm{Hg}$.).

Schoenthal, Gisela (Hg.) (1998): Feministische Linguistik - Linguistische Geschlechterforschung: Ergebnisse Konsequenzen Perspektiven. (Germanistische Linguistik).

Schröter, Beate (1994): Die Sprache der DDR im Spiegel ihrer Literatur. Berlin.

Schweizerische Bundeskanzlei (1996): Leitfaden zur sprachlichen Gleichbehandlung. Bern.

SPRACHE MACHT POLITIK (1994): Wie die ausschließlich weiblichen Personenbezeichnungen die Gemeindeordnung von Wädenswil zu Fall brachten. Herausgeberinnen: Fachstelle für Gleichberechtigungsfragen des Kantons 
Zürich. Büro für die Gleichstellung von Frau und Mann des Kantons BaselLandschaft. Fachstelle für Frauenenfragen der Stadtverwaltung Zürich. Zürich.

Stickel, Gerhard (1988): Beantragte Regelungen zur ,sprachlichen Gleichbehandlung“. Darstellung und Kritik. In: ZGL 16, S. 330-355.

Trempelmann, Gisela (1990): Die kleinste Menschin der Welt. In: Sprachpflege und Sprachkultur 2, 1990, S. 36-39.

Trempelmann, Gisela (1998): Leserinnen/LeserInnen Ost wie West. Zu Bezeichnungen und Anredeformen in den östlichen Bundesländern. In: Schoenthal $(\mathrm{Hg}$.).

Wegner, Hildeburg/Köhler, Hanne/Kopsch, Cordelia (1990): Frauen fordern eine gerechte Sprache. Gütersloh.

Wetschanov, Karin (1995): „Als wenns a Grammatikfehler wär": Splittingverhalten einer Gruppe mit links-alternativ feministischer Uni-Sozialisation. Diplomarbeit, Wien.

Wodak, Ruth/Feistritzer, Gert/Moosmüller, Silvia/Doleschal, Ursula (1987): Sprachliche Gleichbehandlung von Frau und Mann. Linguistische Empfehlungen zur sprachlichen Gleichbehandlung von Frau und Mann im öffentlichen Bereich. Hrsg. vom Bundesministerium für Arbeit und Soziales. Wien.

Zimmer, Dieter E. (1997): Deutsch und anders. Die Sprache im Modernisierungsfieber. Reinbek. 


\section{Anhang}

Text 1

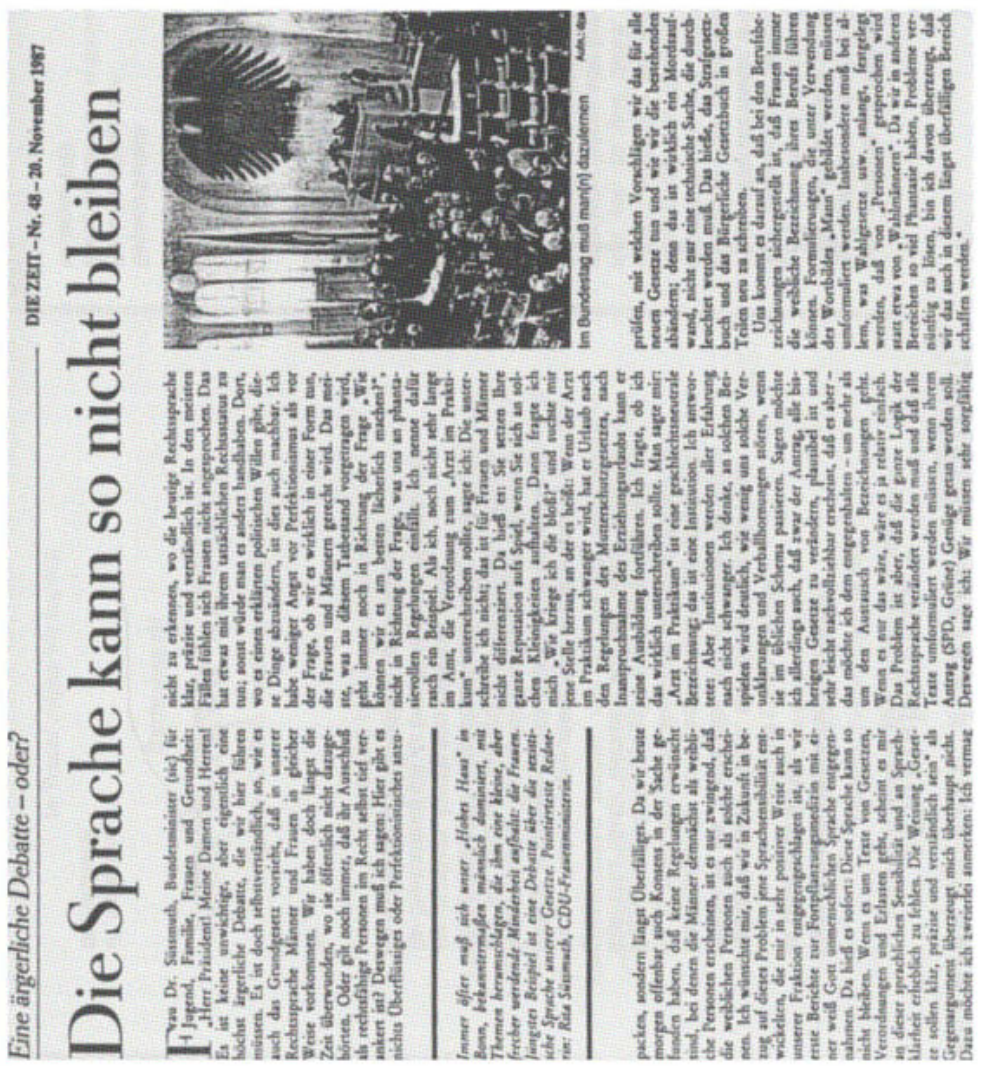



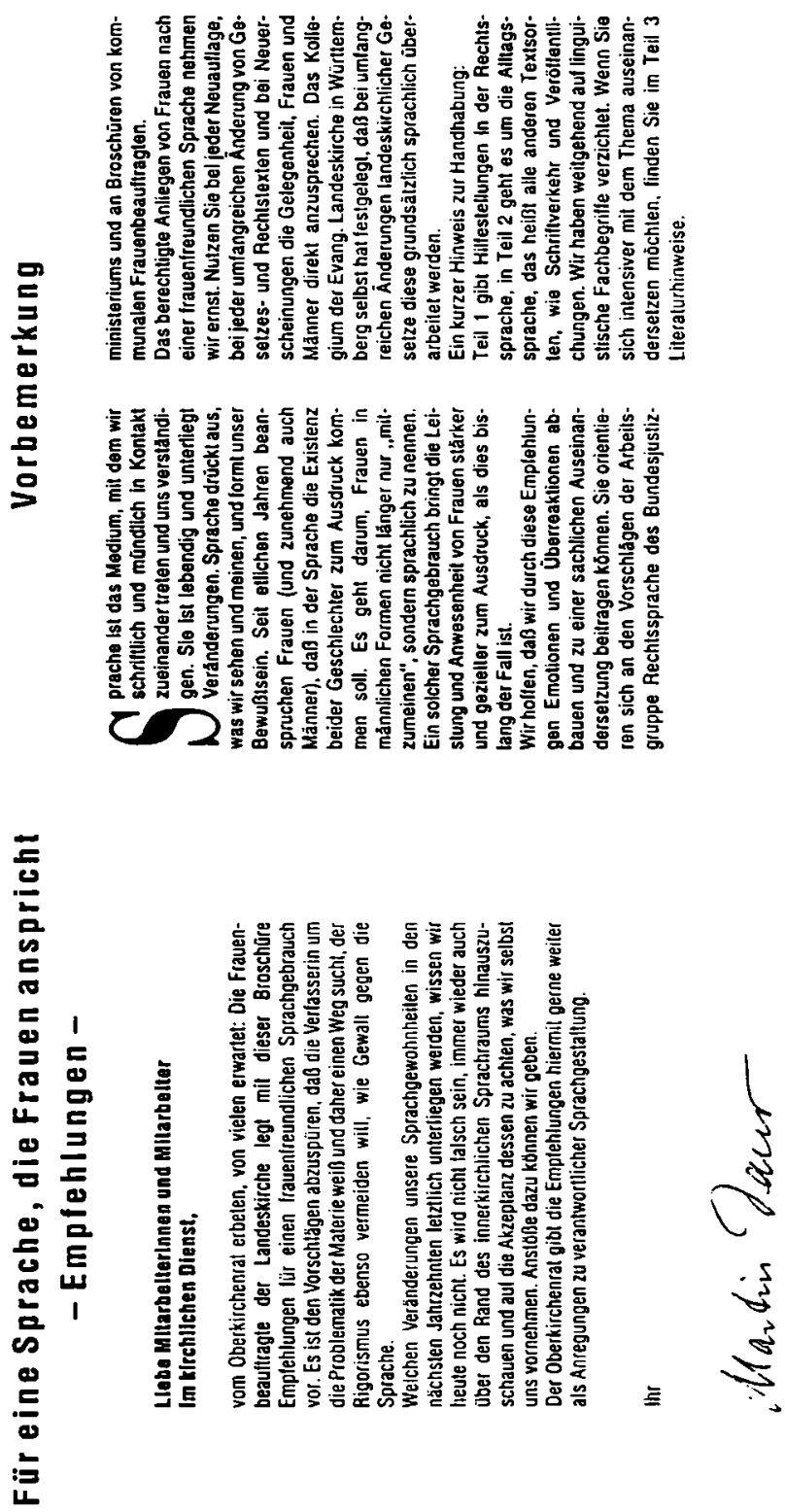

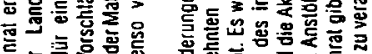

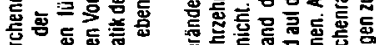

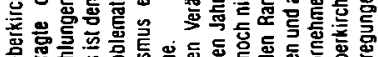

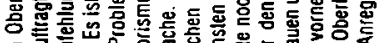

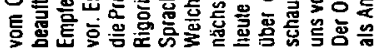
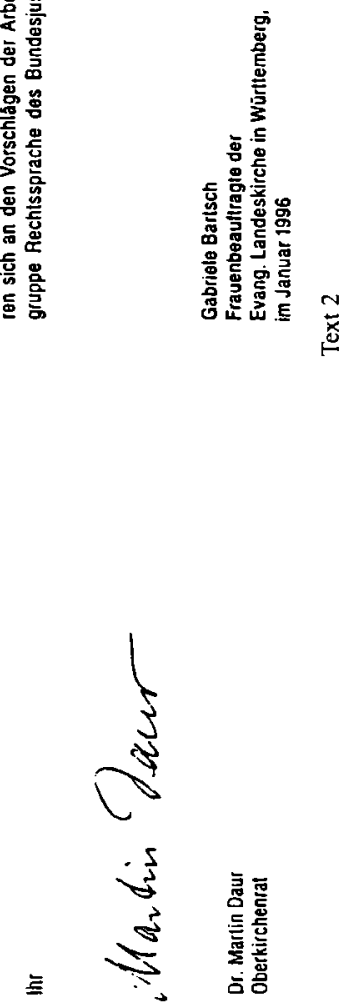

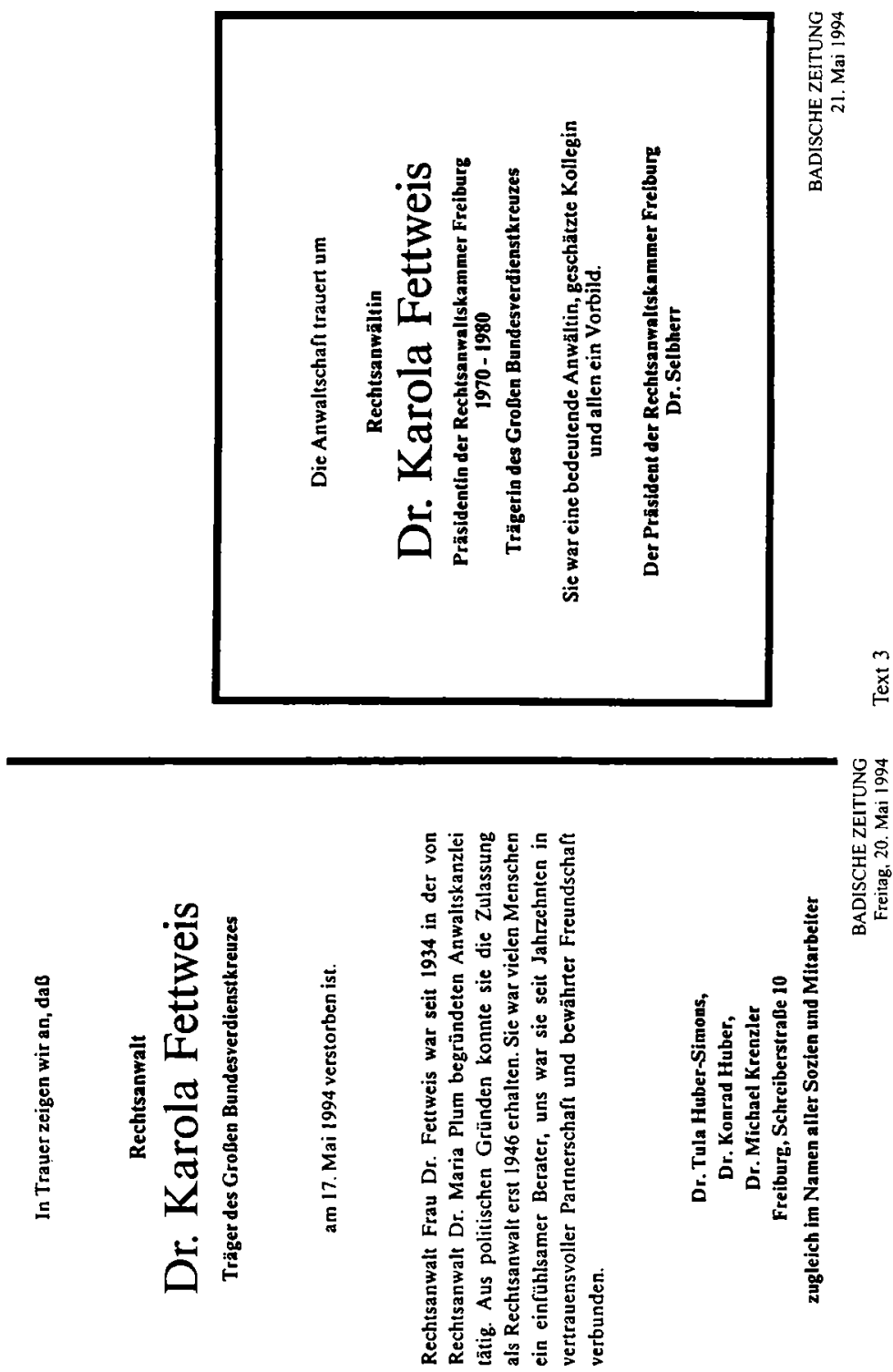


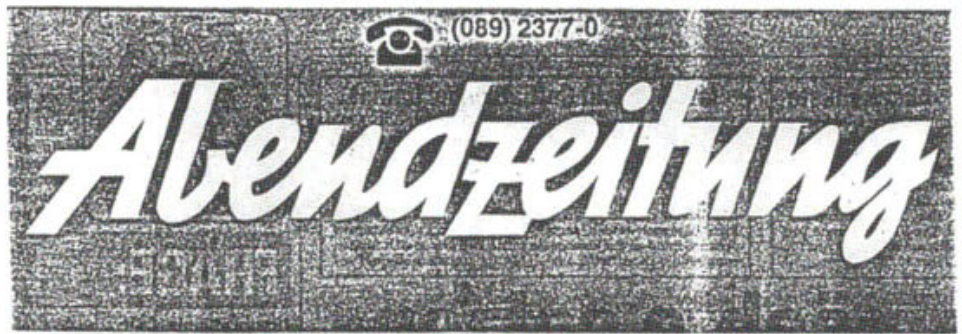

7 Pfennin Mt.275/48 Mittwoch, 28. November 1998

Postiach 200104,8 Münchies Os 10, * L L 1200 * * Dis 12,
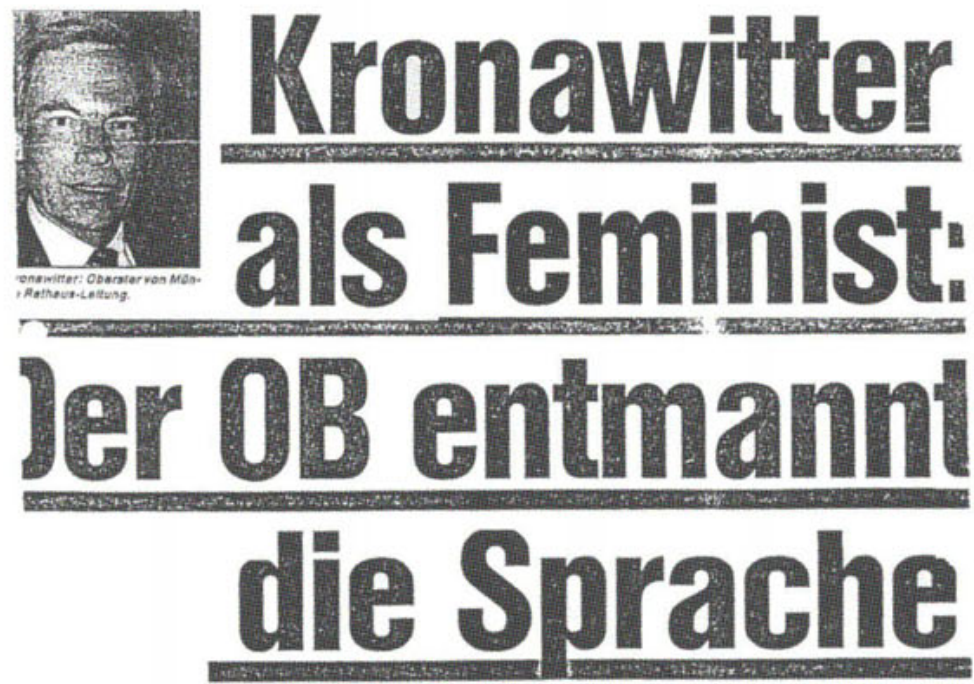

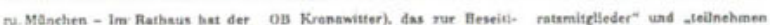
Frminismus einen acheren Schlag gumg der sprachiliches Ungieichbe. Personen". Avs dem "Leiter" wi

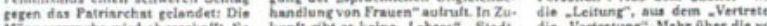

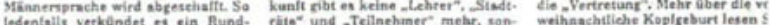

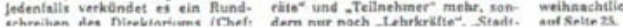

Text 4 